\title{
Risk of cervical intra-epithelial neoplasia and invasive cancer of the cervix in DES daughters
}

\author{
J. Verloop ${ }^{\text {a }}$, F.E. van Leeuwen ${ }^{\text {a }}$, Th.J.M. Helmerhorst ${ }^{\text {b }}$, I.M.C.M. de Kok ${ }^{\text {c }}$, E.J.M. van Erp ${ }^{\text {d }}$, \\ H.H. van Boven ${ }^{\text {e }}$, M.A. Rookus ${ }^{\mathrm{a}, *}$ \\ a Department of Epidemiology, Netherlands Cancer Institute, Amsterdam, The Netherlands \\ b Department of Obstetrics and Gynaecology, Erasmus MC, University Hospital Rotterdam, The Netherlands \\ c Department of Public Health, Erasmus MC, The Netherlands \\ d De Praktijk, Utrecht, The Netherlands \\ e Department of Pathology, Netherlands Cancer Institute, Amsterdam, The Netherlands
}

\section{H I G H L I G H T S}

- Information on CIN was based on the nationwide pathology registry.

- No increased risk for CIN2 + was found among DES daughters.

- DES daughters with DES-related malformations had higher rates of CIN1 probably due to screening.

\section{A R T I C L E I N F O}

\section{Article history:}

Received 22 August 2016

Received in revised form 23 November 2016

Accepted 29 November 2016

Available online $\mathrm{xxxx}$

\section{Keywords:}

Diethylstilbestrol

Squamous neoplasia

Cervical dysplasia

Cervix

Pap smear screening

\begin{abstract}
A B S T R A C T
Objective. Women exposed to diethylstilbestrol in utero (DES) have an increased risk of clear cell adenocarcinoma (CCA) of the vagina and cervix, while their risk of non-CCA invasive cervical cancer is still unclear.

Methods. We studied the risk of pre-cancerous (CIN) lesions and non-CCA invasive cervical cancer in a prospective cohort of 12,182 women with self-reported DES exposure followed from 2000 till 2008. We took screening behavior carefully into account. Incidence was obtained through linkage with the Netherlands Nationwide Pathology database (PALGA). General population data were also derived from PALGA.

Results. The incidence of CIN1 was increased (Standardized Incidence Ratio (SIR) $=2.8,95 \%$ Confidence Interval $(\mathrm{Cl})=2.3$ to 3.4), but no increased risk was observed for CIN2 + (CIN2, CIN3 or invasive cancer) compared to the screened general population ( $\mathrm{SIR}=1.1,95 \% \mathrm{CI}=0.95$ to1.4). Women with DES-related malformations had increased risks of both CIN1 and CIN2 + ( $\mathrm{SIR}=4.1,95 \% \mathrm{CI}=3.0$ to 5.3 and $\mathrm{SIR}=1.5,95 \% \mathrm{CI}=1.1$ to 2.0 , respectively). For CIN2 +, this risk increase was largely restricted to women with malformations who were more intensively screened.

Conclusions. An increased risk of CIN1 among DES daughters was observed, especially in women with DES-related malformations, probably mainly due to screening. The risk of CIN2 + (including cancer) was not increased. However, among DES daughters with DES-related malformations a true small risk increase for non-CCA cervical cancer cannot be excluded.
\end{abstract}

(c) 2016 The Authors. Published by Elsevier Inc. This is an open access article under the CC BY-NC-ND license (http://creativecommons.org/licenses/by-nc-nd/4.0/).

\section{Introduction}

In the late 1940 s to the early 1970 s several millions of pregnant women worldwide received Diethylstilbestrol (DES) in order to prevent miscarriages and other pregnancy complications [1-4]. Next to high risk of clear cell adenocarcinoma of the vagina and cervix (CCA), the well-

* Corresponding author at: Department of Epidemiology, Netherlands Cancer Institute, Plesmanlaan 121, 1066 CX Amsterdam, The Netherlands.

E-mail address: m.rookus@nki.nl (M.A. Rookus). established adverse health effects in the female offspring (DES daughters) include several reproductive tract abnormalities, among which are the presence of glandular tissue (adenosis) and metaplastic squamous epithelium in the vagina and ectocervix. It has been speculated that these epithelial changes might increase the risk of cancer, not only adenocarcinoma, but also squamous cell cancer, and precancerous lesions [5-8]. A two-fold risk of cervical dysplasia (CIN2 +) was observed in the National Cancer Institute's DES Combined Cohort Follow-up Study (NCI DES study), with a 74\% increased risk among DES daughters who had vaginal epithelial changes [7,8]. For invasive 
squamous cell cervical cancer findings were inconclusive [9]. In a previous report of our Dutch DES cohort a (non-statistically significant) decreased risk of invasive squamous cell cervical cancer was found [10].

In this report we examined the risk of cervical intra-epithelial lesions (CIN) and cervical cancer in a large cohort of Dutch DES-daughters, compared to the screened general population. A unique feature of this study is that all outcomes and reference data were obtained from the Netherlands nationwide pathology database (PALGA), which enabled us to account for screening behavior.

\section{Patients and methods}

The DES-net project is a nationwide retrospective cohort study with prospective follow-up among DES daughters in the Netherlands. DES daughters were identified through the registry of the Netherlands DES Center that was established in 1992 in order to deal with future health claims. Documented DES exposure at time of registration was not required. In the period March 2000-December 2004 DES daughters were sent a 16-page self-administered questionnaire about risk factors for hormone-related cancers and medical history (response 63\%, supplementary Fig. 1). In addition to the questionnaire, women granted permission to abstract data from their medical records by means of a written informed consent. Furthermore, women provided us, if available, with a copy of the medical file of their mothers in which DES exposure in utero was confirmed.

\section{Assessment of outcome and screening history}

Detailed information on CIN, invasive cervical cancer and screening history was retrieved from PALGA [11]. PALGA is a nationwide database of excerpts of all histopathology and cytopathology reports made since 1989. The PALGA Surveillance Committee granted us permission to link all study subjects with PALGA (both responders and non-responders to the questionnaire, but refusers (6\%) excluded) under strict privacy procedures. The study was approved by the Institutional Review Board of the Netherlands Cancer Institute.

All CINs and cancer were coded according to the systemized nomenclature of medicine (SNOMED). For each woman the first occurrence of the highest grade of CIN was used in the analysis (supplementary Table 1). CIN1 was defined as mild dysplasia, CIN2 as moderate dysplasia, CIN3 as severe dysplasia or carcinoma in situ and cervical cancer as invasive cervical squamous cell carcinoma and non-CCA adenocarcinoma. CIN2 + was defined as a combination of CIN2, CIN3 and invasive cancer. For each woman the number of episodes, as a proxy for the number of screening rounds, was calculated. By definition, a screening episode started with a primary smear, if necessary followed by secondary smears in case of an abnormal smear or a smear of inadequate quality. An episode ended when follow-up was complete according to the Dutch guidelines (i.e. the dates of the third consecutive negative smears after a primary smear with high grade squamous cell intra-epithelial lesion (HSIL) smear, the second consecutive negative smears after a primary smear with low grade squamous cell intra-epithelial lesion (LSIL), the one consecutive adequate smear after a primary smear of inadequate quality within 6 months, or at the date 4 years after the primary smear when no (adequate) follow-up smears were done. Thus, by definition, post-diagnostic follow-up smears were attributed to the same episode as the diagnosed lesion.

\section{Covariates}

Questionnaire data on DES-related reproductive tract abnormalities (including adenosis, squamous cell metaplasia, transverse vaginal ridges, cockscomb, cervical collars, hoods, pseudo-polyps, hypoplastic cervix, uterine cavum malformations and tubal malformations) were verified by medical file. The term vaginal/cervical epithelial changes (VCEC) is used to refer to adenosis or squamous cell metaplasia of both the vagina and the ecto-cervix (enlarged ectropion). Other covariates (educational level, indication for maternal DES usage, age at first gynaecological DES examination, and number of smears and colposcopies during five years preceding the questionnaire) were based on self-report. Vital status was obtained by linkage with the Netherlands Office of Death Registry (CBG) and updated till October 2007.

\section{Verification of DES exposure}

Documented DES exposure was available for a minority of subjects. Mothers' medical records were hard to trace as archives of hospitals and general practitioners had been destroyed. For a subgroup of participants $(n=115)$ we verified self-reported DES exposure with medical records in four hospitals where all records had been kept [10]. For 76\% of the women DES exposure was confirmed, in 3\% a medicine different from DES was recorded and in $21 \%$ no DES was mentioned in the hospital medical file, while prescription by general practitioner could not be excluded. Because the agreement between self-report and verified DES-exposure was acceptable, we included all women in the analyses, irrespective of whether DES exposure was medically verified.

\section{Statistical analysis}

Follow-up started on January 1st 2000, at age 29 years, whichever was last. We excluded women younger than age 29 because the Dutch general population screening program is restricted to women aged 30-60 years, and women are invited in the year they become 30 years. Follow-up ended at 30th November 2008, the date of first occurrence of intra-epithelial cervical neoplasia (CIN) or cervical cancer, death, date of uterus extirpation/cervix amputation, date of the 65th anniversary, whichever came first. After exclusion of ineligible women ( $n=287$, supplementary Fig. 1$), 11,895$ women $(100,287$ personyears) were left for analyses.

Standardized Incidence Ratios (SIRs) were calculated by comparing the number of observed cases with CIN and invasive cervical cancer in our study with age-, sex- and calendar period-specific numbers from PALGA $[12,13]$. The number of women at risk in the general population was obtained from Statistics Netherlands (www.cbs.nl) and adjusted for women without an uterus (from the Dutch Hospital Discharge database (LMR), based on 5-year age categories (30-64)). To calculate the total number of screened women at risk in the general population we additionally applied 5-year coverage rates per five year age category from the year 2005 [14] (supplementary, Table 2). 95\% confidence intervals (CI) were calculated assuming a Poisson distribution [15].

Because it is generally known that first screens have higher detecting rates than following tests, we excluded prevalent lesions, detected during the first screening episode, both in the general population as the study population. In order to achieve this for the latter population, analyses were restricted to participants who reported to have had a smear during the five years preceding the questionnaire. Furthermore, we calculated SIRs for women stratified according to the number of episodes, the presence of DES-related malformations and attained age. Additionally, we examined which type of examination (biopsy or cytology) and which outcome directly preceded the histological diagnosis of CIN.

We used the Kaplan-Meier method to compare the cumulative incidence of CIN lesions among subgroups of women. Furthermore, Cox regression analysis was performed to calculate hazard ratios (HR) in order to quantify the effect of different covariates on the risk of CIN within the exposed cohort, with adjustment for the other covariates. Both in the Kaplan-Meier and the Cox regression model, age was used as the time metric. All analyses were conducted using STATA release 11 SE. 
Table 1

Characteristics of 11,895 DES daughters and the subgroup of cases diagnosed with cervica intra-epithelial neoplasia (all grades) and cervical cancer during follow-up, 2000-2008.

\begin{tabular}{|c|c|c|c|}
\hline \multirow[t]{2}{*}{ Characteristic } & $\begin{array}{l}\text { All women } \\
(N=11,894)\end{array}$ & \multicolumn{2}{|c|}{$\begin{array}{l}\text { CIN (all grades) and } \\
\text { cervical cancer }^{\mathrm{a}} \\
(N=392)\end{array}$} \\
\hline & $\mathrm{N}(\%)$ & \multicolumn{2}{|l|}{$\mathrm{N}(\%)$} \\
\hline \multicolumn{4}{|l|}{ Year of birth } \\
\hline$\leq 1955$ & $1446(12)$ & \multicolumn{2}{|c|}{$27(7)$} \\
\hline 1956-1960 & $2496(21)$ & \multicolumn{2}{|c|}{$66(17)$} \\
\hline 1961-1965 & $2926(25)$ & \multicolumn{2}{|c|}{$108(27)$} \\
\hline 1966-1970 & 3405 (29) & \multicolumn{2}{|c|}{147 (37) } \\
\hline$>1970$ & & $44(11$ & \\
\hline Age at end of follow up & & & \\
\hline $30-40 y$ & & $117(3$ & \\
\hline $6088(51)$ & & $216(5$ & \\
\hline $2682(23)$ & & $59(15$ & \\
\hline Year of registration & & & \\
\hline $11,009(9$ & & $357(9$ & \\
\hline 1993-1999 & & $13(3)$ & \\
\hline 2000-2005 & & $22(6)$ & \\
\hline Questionnaire completed & & & \\
\hline Yes & & $263(6$ & \\
\hline $4416(37)$ & & $129(3$ & \\
\hline $\begin{array}{l}\text { Number of screening episodes } \\
\text { during follow-up }{ }^{\mathrm{b}}\end{array}$ & & & \\
\hline 0 episodes & & $0(0)$ & \\
\hline 1-2 episodes & & $128(3$ & \\
\hline 3-5 episodes & & $189(4$ & \\
\hline$>5$ episodes & & $75(18$ & \\
\hline Number of cervical/vaginal smears & & & \\
\hline Cervical smears & & & \\
\hline $2091(18)$ & & $3(1)$ & \\
\hline $4400(37)$ & & $27(7)$ & \\
\hline $3496(29)$ & & $112(2$ & \\
\hline $1907(16)$ & & $250(6$ & \\
\hline Vaginal smears & & & \\
\hline $8866(75)$ & & $217(5$ & \\
\hline $1148(10)$ & & $43(11$ & \\
\hline $1511(13)$ & & $98(25$ & \\
\hline $369(3)$ & & $34(9)$ & \\
\hline Number of biopsies & & & \\
\hline Cervical biopsies & & & \\
\hline $11,023(9$ & & $0(0)$ & \\
\hline $584(5)$ & & $151(3$ & \\
\hline $287(2)$ & & $241(6$ & \\
\hline Vaginal biopsies & & & \\
\hline $11,793(9$ & & $372(9$ & \\
\hline $101(1)$ & & $20(5)$ & \\
\hline Treatment & & & \\
\hline No treatment & & $192(4$ & \\
\hline Biopsy & & $8(2)$ & \\
\hline Lis excision, conisation & & $178(4$ & \\
\hline Resection, extirpation & & $14(4)$ & \\
\hline & $\begin{array}{l}\text { All } \\
\text { won }\end{array}$ & nen & $\begin{array}{l}\text { CIN (all grades) and } \\
\text { cervical cancer }\end{array}$ \\
\hline Women with questionnaire only & $N=$ & 7478 & $N=263$ \\
\hline DES confirmation & & & \\
\hline Medical file mother & 871 & $(12)$ & $36(14)$ \\
\hline $\begin{array}{l}\text { DES-related reproductive tract abnormalities } \\
\text { (VCEC and structural abnormalities) }\end{array}$ & 1630 & $0(22)$ & $87(33)$ \\
\hline $\begin{array}{l}\text { History of frequent vaginal screening (before } \\
\text { age } 50 \text { ) or frequent cervical screening before } \\
\text { age 30) }\end{array}$ & 1953 & $3(26)$ & $82(31)$ \\
\hline No information available & 3024 & $4(40)$ & $58(22)$ \\
\hline Year of first gynaecological examination ${ }^{c}$ & & & \\
\hline No DES-related gynaecological examination & 1396 & $6(19)$ & $36(14)$ \\
\hline$<1992$ & 4638 & $8(62)$ & $178(68)$ \\
\hline 1992-1999 & 891 & $(12)$ & $33(12)$ \\
\hline$>2000$ & 23( & & $2(1)$ \\
\hline Missing & 530 & (7) & $14(5)$ \\
\hline Age at first gynaecological examination ${ }^{c}$ & & & \\
\hline No DES-related gynaecological examination & 1396 & $6(19)$ & $36(14)$ \\
\hline$<20$ & 2425 & $5(32)$ & $111(42)$ \\
\hline $20-29$ & 2402 & $2(32)$ & $84(32)$ \\
\hline $30+$ & 725 & $(10)$ & $18(7)$ \\
\hline
\end{tabular}

Table 1 (continued)

\begin{tabular}{|c|c|c|}
\hline Women with questionnaire only & $\begin{array}{l}\text { All } \\
\text { women } \\
N=7478\end{array}$ & $\begin{array}{l}\text { CIN (all grades) and } \\
\text { cervical cancer } \\
N=263\end{array}$ \\
\hline Missing & $530(7)$ & $14(5)$ \\
\hline \multicolumn{3}{|l|}{ Medical indication DES use mother } \\
\hline Both threatened and habitual abortion & $1289(17)$ & $50(19)$ \\
\hline Threatened abortion only & $1879(25)$ & $58(22)$ \\
\hline Habitual abortion only & $3790(51)$ & $137(52)$ \\
\hline Other reason/unknown & $520(7)$ & $18(7)$ \\
\hline \multicolumn{3}{|l|}{ Highest educational level } \\
\hline Primary school & $939(13)$ & $31(12)$ \\
\hline Secondary school & $3551(47)$ & $123(47)$ \\
\hline College or university & $2835(38)$ & $100(38)$ \\
\hline Unknown or missing & $153(2)$ & $9(3)$ \\
\hline \multicolumn{3}{|c|}{$\begin{array}{l}\text { Number of Pap smears during } 5 \text { years preceding } \\
\text { questionnaire }\end{array}$} \\
\hline No smears & $431(6)$ & $4(2)$ \\
\hline $1-2$ & $2646(35)$ & $67(25)$ \\
\hline$>3$ & $4030(54)$ & $177(67)$ \\
\hline Unknown or missing & $371(5)$ & $15(6)$ \\
\hline \multicolumn{3}{|l|}{$\begin{array}{l}\text { Number of colposcopies during } 5 \text { years } \\
\text { preceding questionnaire }\end{array}$} \\
\hline No colposcopies & $1829(24)$ & $48(18)$ \\
\hline $1-2$ & $2236(30)$ & $91(35)$ \\
\hline$>3$ & $1168(16)$ & $75(29)$ \\
\hline Unknown or missing & $2245(30)$ & $49(18)$ \\
\hline \multicolumn{3}{|l|}{ Medically verified DES-related malformations ${ }^{\mathrm{d}}$} \\
\hline Not reported & $5321(71)$ & $154(59)$ \\
\hline Adenosis, squamous neoplasia (VCEC) & $1347(18)$ & $79(30)$ \\
\hline Structural anomalies & $555(7)$ & $22(8)$ \\
\hline Not specified & $205(3)$ & $7(3)$ \\
\hline Missing & $50(1)$ & $1(0)$ \\
\hline
\end{tabular}

a $\mathrm{CIN}=$ cervical intra-epithelial lesion; $\mathrm{VCEC}=$ vaginal/cervical epithelial changes.

b Screening episodes defined as primary smear and, if necessary, followed by secondary smears in case of an abnormal smear of a smear of inadequate quality.

c Gynaecological examination (any of these): palpation, colposcopy, cervical smears and/or vaginal smears.

d 813 women with both VCEC and structural abnormalities were categorized as VCEC. VCEC included cervical and vaginal adenosis and squamous cell metaplasia. Structural abnormalities included transverse vaginal ridges, cockscomb, cervical collars, hoods, pseudopolyps, hypoplastic cervix, uterine cavum malformations and tubal malformations.

\section{Results}

Among the 11,894 women in our analytic cohort, 392 women (3\%) had a histologically confirmed CIN lesion or cervical cancer detected during follow-up (Table 1). Fifteen women had two lesions (same or different grade) at different time points during follow-up (results not shown). The number of screening episodes was remarkably high among cases; 66\% had more than two episodes during follow-up compared to $42 \%$ in the total DES-exposed cohort. In addition, and also highly correlated to the number of episodes, women with CIN or invasive cancer had undergone a higher number of smears and biopsies compared to the total cohort (>5 cervical smears: $64 \%$ and $16 \%$, respectively). Seventy-four percent of the cases reported to have had their first gynaecological examination before age 30 compared to $64 \%$ of the total cohort. The majority of women had had a Pap smear in the five years preceding the questionnaire, with the number of colposcopies being considerably higher among cases (64\% versus $46 \%$ in the total group). Documented DES exposure was available for 871 women among whom were 36 cases. The higher proportion of cases with DES documentation was probably caused by their search for these records after diagnosis of CIN/invasive cancer or DES-related malformations. Cases more often had DES-related malformations compared to the entire cohort, of which VCEC occurred most frequently (32\% and $20 \%$ for cases and entire cohort, respectively).

Table 2 describes the risk of CIN and cervical cancer among DES daughters compared to the screened female population. For CIN1 lesions an increased risk was observed ( $\mathrm{SIR}=2.8,95 \% \mathrm{CI}=2.3$ to 3.4$)$. SIR estimates for CIN2 and CIN3 were above one but not statistically significant and for invasive cervical cancer a decreased risk (SIR $=0.29$, 
Table 2

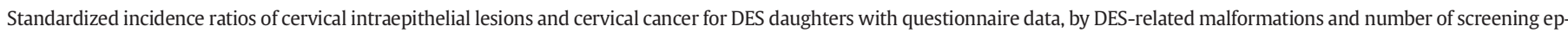
isodes, follow-up period 2000-2008.

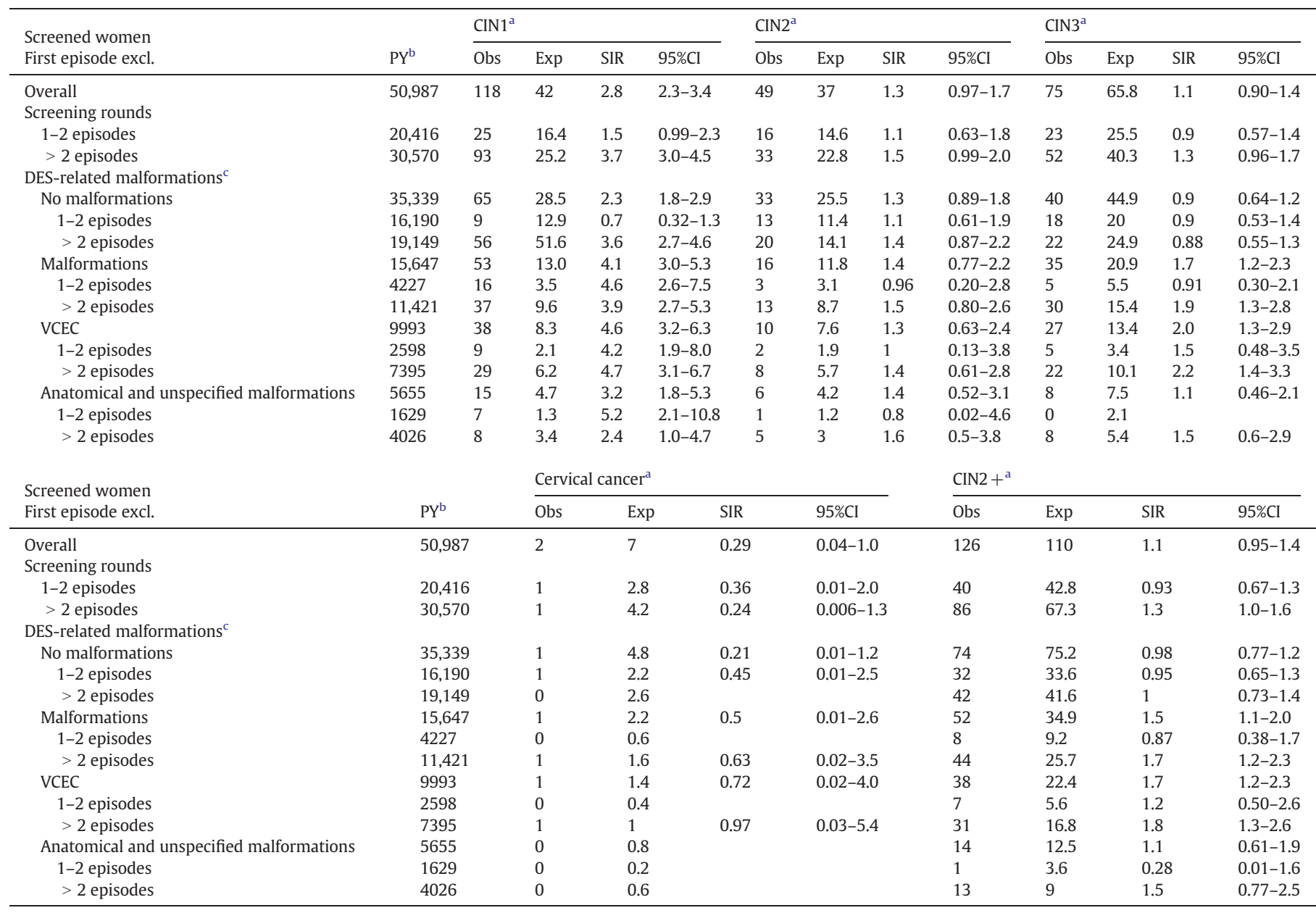

a CIN: cervical intra-epithelial neoplasia. CIN1 (grade 1): Mild dyskaryosis CIN2 (grade 2): moderate dyskaryosis CIN3 (grade 3): severe dyskaryosis/dysplasia or carcinoma in situ. CIN2 + is including cancer. Cancer morphology: 1 squamous cell carcinoma, 1 adenocarcinoma.

b $\mathrm{PY}=$ person years, Obs = observed, Exp = expected, SIR = Standardized incidence ratio, defined as observed number of cancers compared to the expected number of cancers in the general population with the same age; $95 \% \mathrm{CI}=95 \%$ confidence interval based on a Poisson distribution.

c Definition (medically verified) DES-related malformations: Anatomical malformations: transverse vaginal ridges, cockscomb, cervical collars, hoods, pseudo-polyps, hypoplastic

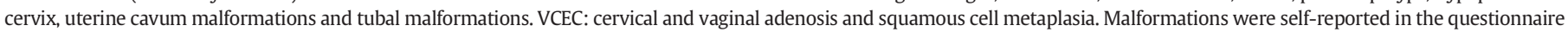
(at start of the follow-up) and validated by medical file.

Table 3

Risk of cervical dysplasia and cancer among screened DES daughters by attained age (exclusion of lesions diagnosed at first screening episode).

\begin{tabular}{|c|c|c|c|c|c|c|c|c|c|}
\hline \multirow{3}{*}{$\begin{array}{l}\text { All PYa } \\
\text { Type of } \\
\text { lesion }^{b}\end{array}$} & \multicolumn{4}{|c|}{ Attained age $<40^{\mathrm{d}}$} & \multicolumn{4}{|c|}{ Attained age $40+^{d}$} & \multirow[b]{3}{*}{$\begin{array}{l}\mathrm{p} \\
\text { difference }^{\mathrm{c}}\end{array}$} \\
\hline & \multicolumn{4}{|c|}{24,345} & \multicolumn{4}{|c|}{26,642} & \\
\hline & $\mathrm{Obs}^{\mathrm{a}}$ & Exp & SIR & $95 \% \mathrm{CI}$ & Obs & Exp & SIR & $95 \% \mathrm{CI}$ & \\
\hline CIN1 & 65 & 20.4 & 3.2 & $2.4-4.1$ & 53 & 21.2 & 2.5 & $1.9-3.3$ & 0.19 \\
\hline CIN2 & 27 & 20.1 & 1.3 & $0.88-2.0$ & 22 & 17.2 & 1.3 & $0.80-1.9$ & 0.86 \\
\hline CIN3 & 48 & 38 & 1.3 & $0.93-1.7$ & 27 & 27.8 & 0.97 & $0.64-1.4$ & 0.27 \\
\hline $\begin{array}{l}\text { Cervical } \\
\text { cancer }\end{array}$ & 1 & 3 & 0.33 & $0.01-1.8$ & 1 & 4 & 0.25 & $0.01-1.4$ & 0.85 \\
\hline CIN2 + & 76 & 61.1 & 1.2 & $0.98-1.6$ & 50 & 49 & 1 & $0.76-1.4$ & 0.28 \\
\hline
\end{tabular}

${ }^{\mathrm{a}} \mathrm{PY}=$ person years, Obs $=$ observed, $\mathrm{Exp}=$ expected, SIR $=$ Standardized incidence ratio, defined as observed number of cancers compared to the expected number of cancers in the general population Cancer morphology: 1 squamous cell carcinoma, 1 adenocarcinoma.

b CIN: cervical intra-epithelial neoplasia. CIN1 (grade 1): Mild dyskaryosis CIN2 (grade 2): moderate dyskaryosis CIN3 (grade 3): severe dyskaryosis/dysplasia or carcinoma in situ. CIN2 + is including cancer. Cancer morphology: 1 squamous cell carcinoma, 1 adenocarcinoma.

c $\mathrm{p}$ difference based on Poisson distribution.

d attained age = age at diagnosis of (first, maximum) CIN or age at end follow-up (30th November 2008).
$95 \% \mathrm{CI}=0.04$ to 1.0 ) was observed. No increased risk for CIN2 + was found ( $\mathrm{SIR}=1.1,95 \% \mathrm{CI}=0.95$ to 1.4$)$. Among women with more than two screening episodes the risks for both CIN1 and CIN2+ were increased $(\mathrm{SIR}=3.7,95 \% \mathrm{CI}=3.0$ to 4.5 and $\mathrm{SIR}=1.3,95 \%$ $\mathrm{CI}=1.0$ to 1.6 , respectively). DES daughters with one to two episodes (suggestive of following the 5-yearly general population cervical screening program) had a less pronounced increased risk of CIN1 ( $\mathrm{SIR}=1.5,95 \% \mathrm{CI}=0.99$ to 2.3$)$ and the risk of $\mathrm{CIN} 2+(\mathrm{SIR}=0.93$, $95 \% \mathrm{CI}=0.67$ to 1.3 ) was no longer increased. Among women with DES-related malformations the risks of CIN1 and CIN2 + were both elevated compared to the screened general population, with the risk increases being most pronounced in the VCEC-group (SIR = 4.6, $95 \% \mathrm{CI}=3.2$ to 6.3 and $\mathrm{SIR}=1.7,95 \% \mathrm{CI}=1.2$ to 2.3 for CIN1 and CIN2 +, respectively).

The SIRs did not vary according to attained age, but numbers were small (Table 3). The risk of CIN1 seemed to be slightly higher among women younger than 40 than at older ages. To study whether the high number of histologically verified CIN1 lesions might be the result of more invasive diagnostic methods we examined which type of examination and outcome preceded the histological diagnosis (Table 4). Half of the CIN1 lesions were preceded by a smear with borderline or mild dyskaryosis which was comparable to general population figures. A 
Table 4

Preceding primary test results in relation to histologically confirmed lesions for 393 DES daughters with CIN lesions and (invasive) cervical cancer.

\begin{tabular}{|c|c|c|c|c|c|}
\hline \multirow[b]{2}{*}{ Result of primary test } & \multicolumn{4}{|c|}{ Histologically confirmed lesion ${ }^{\mathrm{a}}$} & \multirow[b]{2}{*}{ Total N(\%) } \\
\hline & Cancer N(\%) & CIN3 N(\%) & CIN2 N(\%) & CIN1 N(\%) & \\
\hline \multicolumn{6}{|l|}{ Smears ${ }^{\mathrm{b}}$} \\
\hline Cancer & $0(0 \%)$ & $6(5 \%)$ & $0(0 \%)$ & $0(0 \%)$ & $6(2 \%)$ \\
\hline Carcinoma in situ & $1(20 \%)$ & $11(9 \%)$ & $0(0 \%)$ & $2(1 \%)$ & $14(4 \%)$ \\
\hline Severe dyskaryosis (HSIL) & $1(20 \%)$ & $34(26 \%)$ & $9(10 \%)$ & $4(2 \%)$ & $48(12 \%)$ \\
\hline Moderate dyskaryosis (HSIL) & $0(0 \%)$ & $35(27 \%)$ & $23(26 \%)$ & $13(8 \%)$ & $71(18 \%)$ \\
\hline Mild dyskaryosis (LSIL) & $0(0 \%)$ & $22(17 \%)$ & $27(30 \%)$ & $45(27 \%)$ & $94(24 \%)$ \\
\hline Borderline dyskaryosis/ASCUS & $0(0 \%)$ & $16(12 \%)$ & $18(20 \%)$ & $44(26 \%)$ & $78(20 \%)$ \\
\hline \multicolumn{6}{|l|}{ Biopsies } \\
\hline Cancer & $3(60 \%)$ & $0(0 \%)$ & $0(0 \%)$ & $0(0 \%)$ & $3(1 \%)$ \\
\hline CIN3 & $0(0 \%)$ & $3(2 \%)$ & $0(0 \%)$ & $0(0 \%)$ & $3(1 \%)$ \\
\hline CIN2 & $0(0 \%)$ & $2(2 \%)$ & $11(12 \%)$ & $0(0 \%)$ & $13(3 \%)$ \\
\hline CIN1 & $0(0 \%)$ & $0(0 \%)$ & $1(1 \%)$ & $61(36 \%)$ & $62(16 \%)$ \\
\hline Total & 5 & 129 & 89 & 169 & 392 \\
\hline
\end{tabular}

a CIN: cervical intra-epithelial neoplasia. CIN1 (grade 1): Mild dyskaryosis CIN2 (grade 2): moderate dyskaryosis CIN3 (grade 3): severe dyskaryosis/dysplasia or carcinoma in situ. Cancer morphology: 2 squamous cell carcinoma, 2 adenocarcinoma 1 adenosquamous cell carcinoma.

${ }^{\mathrm{b}} \mathrm{HSIL}=$ High grade squamous intraepithelial lesion, LSIL = Lowgrade squameuze intra-epitheliale lesion, ASCUS = atypical squamous cells of undetermined significance.

remarkable finding was the high proportion of cases with CIN1 that were preceded by biopsies without a preceding smear (36\%), which might be indicative of biopsies directed by abnormal colposcopic findings or because of complaints.

The number of episodes was an important predictor for detecting a CIN1 lesion, but not as much for a CIN2 + lesion $\left(\mathrm{HR}_{\mathrm{adj}}=1.99,95 \%\right.$ $\mathrm{CI}=1.24$ to 3.11 and $\mathrm{HR}=1.22,95 \% \mathrm{CI}=0.82$ to 1.81 , respectively)(Table 5, Fig. 1a). Rather, the risk of CIN2 + lesions was slightly increased for women with a history of VCEC or DES-related anatomical abnormalities, though not statistically significantly so $\left(\mathrm{HR}_{\mathrm{adj}}=1.47,95 \% \mathrm{CI}=0.96\right.$ to 2.27 and $\mathrm{HR}=1.52,95 \% \mathrm{CI}=0.91$ to 2.53, respectively, see also Fig. $1 \mathrm{~b})$.

\section{Discussion}

In this study we comprehensively evaluated the risk of CIN and nonCCA cervical cancer among DES daughters with complete follow-up and based on medically verified outcome data. Whereas for low-grade CIN lesions (CIN1) a statistically significantly increased risk was observed in DES daughters compared to the screened general population, the overall risk of CIN2 + (CIN2/3 or invasive cancer) was not increased. However, in women with DES-related malformations increased risks of both CIN1 and CIN2 + were found, with the increased risks being most pronounced in women with VCEC who had been intensively screened.

Table 5

Univariate and multivariate Hazard Ratios for different risk factors for CIN and cervical cancer.

\begin{tabular}{|c|c|c|c|c|c|c|c|c|}
\hline \multirow[b]{3}{*}{ Screened women, exclusion of the first episode } & \multicolumn{4}{|c|}{ CIN1 ${ }^{\mathrm{a}}$} & \multicolumn{4}{|c|}{ CIN2 plus ${ }^{\mathrm{a}}$} \\
\hline & \multicolumn{2}{|c|}{ Univariate } & \multicolumn{2}{|c|}{ Multivariate $^{\mathrm{e}}$} & \multicolumn{2}{|c|}{ Univariate } & \multicolumn{2}{|c|}{ Multivariate $^{\mathrm{b}}$} \\
\hline & HR & $95 \% \mathrm{CI}$ & HR & $95 \% \mathrm{CI}$ & HR & $95 \% \mathrm{CI}$ & HR & $95 \% \mathrm{CI}$ \\
\hline \multicolumn{9}{|l|}{ Episodes $^{\mathrm{b}}$} \\
\hline $1-2$ episodes & 1.00 & Reference & 1.00 & Reference & 1.00 & Reference & 1.00 & Reference \\
\hline$>2$ episodes & 2.43 & $1.56-3.79$ & 1.99 & $1.24-3.11$ & 1.31 & $0.90-1.9$ & 1.22 & $0.82-1.81$ \\
\hline \multicolumn{9}{|l|}{ DES-related malformations ${ }^{c}$} \\
\hline No malformations & 1.00 & Reference & 1.00 & Reference & 1.00 & Reference & 1.00 & Reference \\
\hline VCEC & 1.90 & $1.26-2.84$ & 1.38 & $0.90-2.12$ & 1.68 & $1.13-2.49$ & 1.47 & $0.96-2.27$ \\
\hline Structural abnormalities & 1.22 & $0.69-2.16$ & 1.05 & $0.59-1.86$ & 1.49 & $0.90-2.46$ & 1.52 & $0.91-2.53$ \\
\hline Not specified & 1.66 & $0.66-4.13$ & 1.24 & $0.50-3.13$ & 0.58 & $0.14-2.38$ & 0.56 & $0.14-2.32$ \\
\hline Primary school & 1.00 & Reference & 1.00 & Reference & 1.00 & Reference & 1.00 & Reference \\
\hline Secondary school & 0.84 & $0.48-1.47$ & 0.81 & $0.46-1.42$ & 1.18 & $0.64-2.2$ & 1.19 & $0.64-2.21$ \\
\hline College or university & 0.79 & $0.44-1.41$ & 0.75 & $0.42-1.34$ & 1.26 & $0.67-2.37$ & 1.24 & $0.66-2.33$ \\
\hline Unknown or missing & 1.97 & $0.66-5.90$ & 1.94 & $0.65-5.83$ & 1.24 & $0.28-5.54$ & 1.18 & $0.26-5.29$ \\
\hline \multicolumn{9}{|l|}{ Age first gynaecological DES-examination ${ }^{\mathrm{e}}$} \\
\hline No examination & 1.00 & Reference & 1.00 & Reference & 1.00 & Reference & 1.00 & Reference \\
\hline$<20$ & 2.83 & $1.22-6.54$ & 1.71 & $0.71-4.11$ & 0.76 & $0.45-1.31$ & 0.44 & $0.23-0.80$ \\
\hline $20-29$ & 2.64 & $1.16-5.97$ & 1.63 & $0.69-3.83$ & 0.60 & $0.34-1.03$ & 0.34 & $0.19-0.63$ \\
\hline $30+$ & 2.02 & $0.78-5.40$ & 1.29 & $0.47-3.51$ & 0.45 & $0.18-1.13$ & 0.26 & $0.10-0.69$ \\
\hline Missing & 1.39 & $0.44-4.42$ & 0.98 & $0.30-3.18$ & 0.56 & $0.24-1.33$ & 0.36 & $0.15-0.88$ \\
\hline \multicolumn{9}{|c|}{ Number of colposcopies ( 5 year preceding questionnaire) ${ }^{d}$} \\
\hline No colposcopies & 1.00 & Reference & 1.00 & Reference & 1.00 & Reference & 1.00 & Reference \\
\hline $1-2$ & 1.16 & $0.69-1.96$ & 0.95 & $0.55-1.61$ & 1.36 & $0.82-2.25$ & 1.53 & $0.90-2.60$ \\
\hline$>3$ & 2.09 & $1.23-3.54$ & 1.46 & $0.84-2.54$ & 1.92 & $1.13-3.27$ & 2.13 & $1.19-3.81$ \\
\hline Unknown or missing & 0.65 & $0.35-1.20$ & 0.64 & $0.35-1.19$ & 0.71 & $0.39-1.30$ & 0.74 & $0.40-1.36$ \\
\hline
\end{tabular}

HR = Hazard ratio.

a CIN: cervical intra-epithelial neoplasia. CIN1 (grade 1), CIN2 + : including CIN2, CIN3 and cervical cancer (CCA excluded).

b Screening episodes defined as primary smear and, if necessary, followed by secondary smears in case of an abnormal smear of a smear of inadequate quality.

c Definition DES-related malformations: Anatomical malformations: transverse vaginal ridges, cockscomb, cervical collars, hoods, pseudo-polyps, hypoplastic cervix, uterine cavum

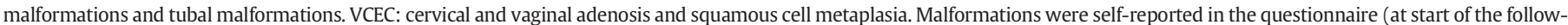
up) and validated by medical file.

d Based on self-report.

e Multivariate analysis: adjusted for all other risk factors. 

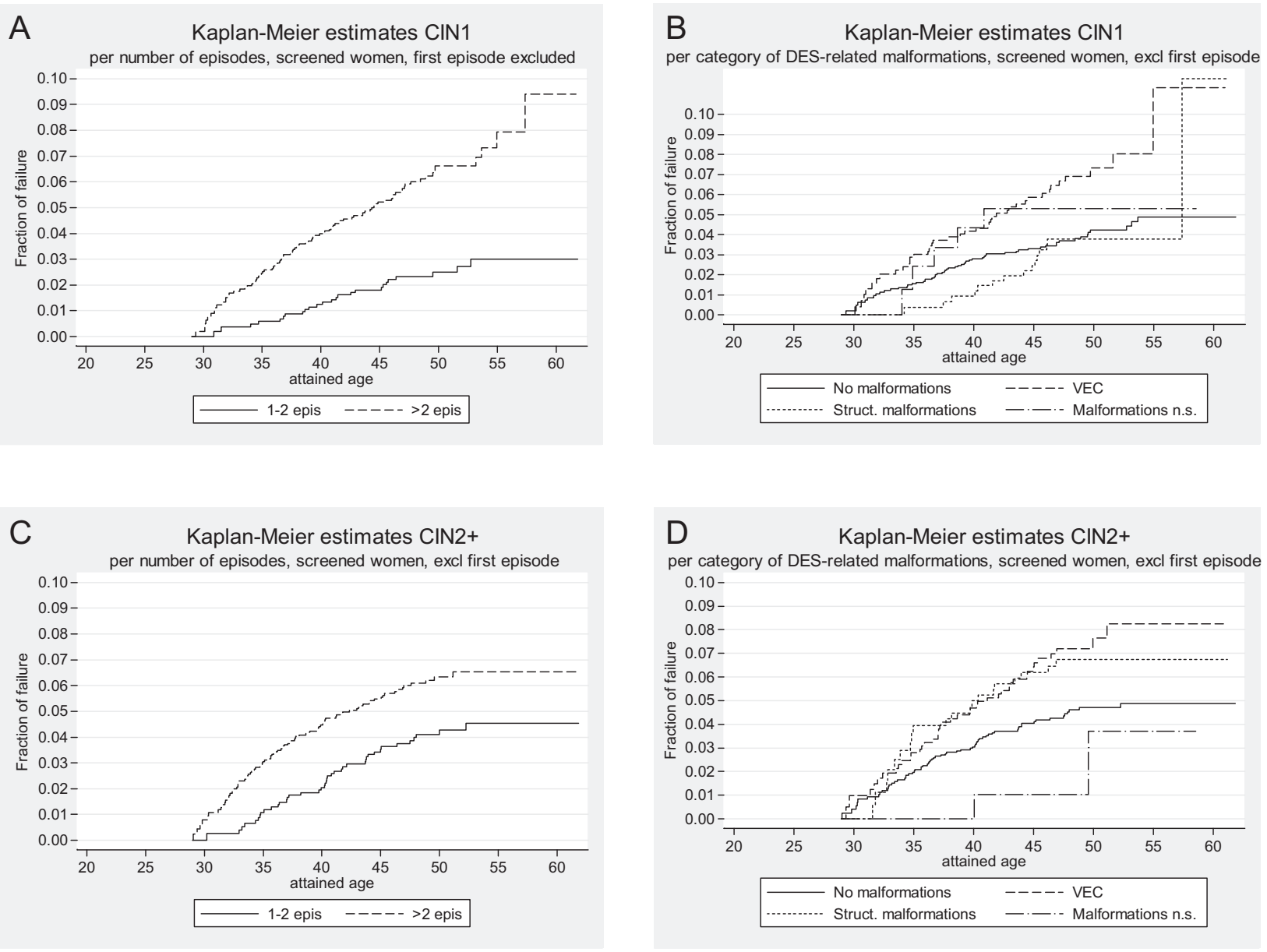

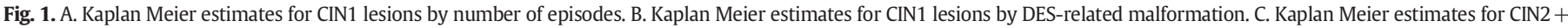
lesions by number of episodes. D. Kaplan Meier estimates for CIN2 + lesions by DES-related malformation.

To our knowledge, the risk of histologically confirmed CIN1 among DES daughters has not been studied before. In 1984, Robboy et al. did examine the risk of mild dysplasia among DES daughters, but with inclusion of lesions that were verified by cytology only [6]. Histological detection of CIN lesions, especially with regard to mild lesions, is shown to vary by differences in diagnostic criteria, follow-up recommendations and referral rates [16]. Also, a high frequency of screening might lead to overdiagnosis of CIN lesions that have a high chance of regression [17-19]. Furthermore, the detection of CIN1 lesions in DES daughters might suffer from histological misclassification due to the common presence of VCEC and a wider transformation zone [20]. Thus, the observed increased risk of CIN1 among DES daughters seems mainly attributable to intensive screening (cytological screening next to screening by regular coloposcopic examinations) as part of the DES screening protocol [21].

Another important finding of our study is that we found no overall increased risk for CIN2 + . This seems in contrast with the NCI DES study [5-8] including 4062 DES-exposed women 1837 unexposed controls, for which a two-fold risk of high grade CIN was reported ( $\mathrm{HR}=1.98,95 \% \mathrm{CI}=1.33$ to 2.94 ) [5-8]. However, a consistent finding was the increased risk of CIN2 + for women with VCEC compared to non-exposed women ( $\mathrm{HR}=2.40,95 \% \mathrm{CI}=1.60$ to 3.61 and $\mathrm{SIR}=$ $1.7,95 \% \mathrm{CI}=1.2$ to 2.3 , in the NCI-study of 2011 (supplementary Table 5) [7] and our study, respectively). Interestingly, when we stratified on screening frequency, the increased risk of CIN2 + in women with VCEC appeared to be restricted to the most intensively screened group. More intensive screening in DES daughters with VCEC (through smears and colposcopic surveillance) may cause overdiagnosis of CIN2 + , not only by detection of lesions that would have regressed spontaneously, but also by possible misinterpretation of VCEC as severe or moderate dyskaryosis. Whether the observed increased risk of CIN2 + is real or an artifact of screening could not be detangled. Most probably, both are true and a real small increased risk of CIN2 + is somewhat enlarged by intensive screening.

There are two reasons why our SIR estimates may be biased to zero, and the estimates of the NCI DES study biased away from the null; the age range included and the data sources used. We excluded women younger than 29 , because our national screening program starts at the year a woman turns 30 years. If the SIR at young ages is higher than later on, our SIR estimate is biased to zero if applied to the entire age range. However, the internal reference group of unexposed controls younger than age 30 in the NCI-DES study may have been screened neither, resulting in bias away from the null. Another important difference between the two studies was the self-reported data on outcome and screening (NCI-DES study) or exposure (DES-net study). We had a relatively small number of women with verified DES-exposure, while the exposure of NCI-DES daughters was $100 \%$ validated. We estimated that at least 76\% of our cohort members was DES-exposed [10]. As a result, our overall risks might be somewhat diluted by inclusion of nonexposed women. However, misclassification of exposure is expected to be minimal in our analysis of women with DES-related malformations (with an attributable risk of 90\% [3,22,23]). Women with self-reported DES-related malformations comprised $36 \%$ of the current analytic cohort. In the NCI-DES cohort the proportion was slightly higher $(45 \%=1864 / 3795)$ [7] which may reflect still some 
misclassification in our cohort, but may also be the result of differences in cumulative DES dosage or timing of DES exposure [24].

In our study complete screening information from PALGA was available. We only missed the colposcopies that were not accompanied by smears. In the NCI-DES cohort health outcomes and screening history were based on self-report. Underreporting or unawareness of abnormal findings by the unexposed group might have biased the results away from zero. Bias in the same direction might result from incomplete adjustment for the screening history [5]. Whereas the use of PALGA data enabled us to adequately control for screening, we could not adjust for confounding. Known risk factors for cervical cancer, like human papilloma virus (HPV) infection, age at first intercourse, number of partners and smoking, may have resulted in confounding [19,25]It is well established that HPV infection is the necessary cause of invasive nonCCA cervical cancer [26]. However, HPV infection alone may not be sufficient and other cofactors, like high parity, smoking and long-term oral contraceptive use, may be necessary for the transition from HPV infection to HSIL/invasive cancer [18]. It is uncertain whether HPV infection plays a role in DES-related tumors like CCA [27]. It has been suggested that the cervical tissue in women with VCEC might be more susceptible to carcinogenic factors like an HPV-infection, which might explain the increased risk estimates of CIN for women with VCEC [5,20]. Another explanation might be that DES daughters have an altered immune system, which renders them more vulnerable to persistent HPV-infection $[28,29]$.

In conclusion, the increased risk of CIN1 among DES daughters, which was most pronounced in women with DES-related malformations, seemed mainly due to intensive screening. The risk of CIN2 + was not increased, suggesting that DES daughters in general do not have an increased risk of cervical cancer (non-CCA) compared to the general population. Among the more frequently screened DES daughters with DES-related malformations overdiagnosis by screening and a potentially small increased risk of CIN2 + could not be disentangled. Our findings underscore the importance of being cautious with respect to invasive diagnostic procedures or treatment since overdiagnosis seem to occur in a group of women which is already strongly medicalized.

\section{Funding}

This study was supported by a grant from the Dutch Cancer Society (grant number 2001-2426) and partially supported by a grant from the Dutch National Institute for Public Health and the Environment (RIVM, grant No 3910010639) (PALEBA data).

\section{Conflict of interest statement}

Drs. L.J.M van Erp is medical advisor of the Dutch DES Fund, which gives compensations to DES victims. The other authors have declared no conflicts of interest.

\section{Acknowledgements}

The authors would like to acknowledge PALGA (Drs. M. Casparie, Dr. L. Overbeek) for providing the follow-up data. The authors thank Corine Penning (Department of Public Health, Erasmus MC) for her comments on the manuscript.

\section{Appendix A. Supplementary data}

Supplementary data to this article can be found online at http://dx. doi.org/10.1016/j.ygyno.2016.11.048.

\section{References}

[1] R.M. Giusti, K. Iwamoto, E.E. Hatch, Diethylstilbestrol revisited: a review of the longterm health effects, Ann. Intern. Med. 122 (1995) 778-788.
[2] O.W. Smith, Diethylstilbestrol in the prevention and treatment of complications of pregnancy, Am. J. Obstet. Gynecol. 56 (1948) 821-834.

[3] S.J. Robboy, R.H. Young, W.R. Welch, G.Y. Truslow, J. Prat, A.L. Herbst, et al., Atypical vaginal adenosis and cervical ectropion. Association with clear cell adenocarcinoma in diethylstilbestrol-exposed offspring. Cancer 54 (1984) 869-875.

[4] A.L. Herbst, H. Ulfelder, D.C. Poskanzer, Adenocarcinoma of the vagina. Association of maternal stilbestrol therapy with tumor appearance in young women, N. Engl. J. Med. 284 (1971) 878-881.

[5] E.E. Hatch, A.L. Herbst, R.N. Hoover, K.L. Noller, E. Adam, R.H. Kaufman, et al., Incidence of squamous neoplasia of the cervix and vagina in women exposed prenatally to diethylstilbestrol (United States), Cancer Causes Control 12 (2001) 837-845.

[6] S.J. Robboy, K.L. Noller, P. O'Brien, R.H. Kaufman, D. Townsend, A.B. Barnes, et al., Increased incidence of cervical and vaginal dysplasia in 3,980 diethylstilbestrol-exposed young women. Experience of the National Collaborative Diethylstilbestrol Adenosis Project, JAMA 252 (1984) 2979-2983.

[7] R.N. Hoover, M. Hyer, R.M. Pfeiffer, E. Adam, B. Bond, A.L. Cheville, et al., Adverse health outcomes in women exposed in utero to diethylstilbestrol, N. Engl. J. Med. 365 (2011) 1304-1314.

[8] R. Troisi, E.E. Hatch, J.R. Palmer, L. Titus, S.J. Robboy, W.C. Strohsnitter, et al., Prenatal diethylstilbestrol exposure and high-grade squamous cell neoplasia of the lower genital tract, Am. J. Obstet. Gynecol. (2016).

[9] R. Troisi, E.E. Hatch, L. Titus-Ernstoff, M. Hyer, J.R. Palmer, S.J. Robboy, et al., Cancer risk in women prenatally exposed to diethylstilbestrol, Int. J. Cancer 121 (2007) 356-360.

[10] J. Verloop, F.E. van Leeuwen, T.J. Helmerhorst, H.H. van Boven, M.A. Rookus, Cancer risk in DES daughters, Cancer Causes Control 21 (2010) 999-1007.

[11] M. Casparie, A.T. Tiebosch, G. Burger, H. Blauwgeers, A. van de Pol, J.H. van Krieken, et al., Pathology databanking and biobanking in the Netherlands, a central role for PALGA, the nationwide histopathology and cytopathology data network and archive, Cell. Oncol. 29 (2007) 19-24.

[12] M. Rebolj, M. van Ballegooijen, E. Lynge, C. Looman, M.L. Essink-Bot, R. Boer, et al., Incidence of cervical cancer after several negative smear results by age 50: prospective observational study, BMJ 338 (2009) b1354.

[13] M. Rebolj, M. van Ballegooijen, L.M. Berkers, D. Habbema, Monitoring a national cancer prevention program: successful changes in cervical cancer screening in the Netherlands, Int. J. Cancer 120 (2007) 806-812.

[14] M. Rebolj, Recent Development in the Dutch Cervical Cancer Screening Programme: Department of Public Health, Erasmus MC, Rotterdam, the Netherlands, 2008.

[15] N.E. Breslow, N.E. Day, Rates and rate standardization, in: N.E. Breslow, N.E. Day (Eds.), Statistical Methods in Cancer Research Volume II - the Design and Analysis of Cohort Studies, Oxford University Press, Oxford 1987, pp. 48-79.

[16] L. Kotaniemi-Talonen, P. Nieminen, M. Hakama, J. Seppanen, J. Ikkala, J. Martikainen, et al., Significant variation in performance does not reflect the effectiveness of the cervical cancer screening programme in Finland, Eur. J. Cancer 43 (2007) 169-174.

[17] A.B. Moscicki, S. Shiboski, N.K. Hills, K.J. Powell, N. Jay, E.N. Hanson, et al., Regression of low-grade squamous intra-epithelial lesions in young women, Lancet 364 (2004) 1678-1683.

[18] X. Castellsague, Natural history and epidemiology of HPV infection and cervical cancer, Gynecol. Oncol. 110 (2008) S4-S7.

[19] M. Schiffman, P.E. Castle, J. Jeronimo, A.C. Rodriguez, S. Wacholder, Human papillomavirus and cervical cancer, Lancet 370 (2007) 890-907.

[20] J. Bornstein, E. Adam, K. Adler-Storthz, R.H. Kaufman, Development of cervical and vaginal squamous cell neoplasia as a late consequence of in utero exposure to diethylstilbestrol, Obstet. Gynecol. Surv. 43 (1988) 15-21.

[21] T.J. Helmerhorst, J.A. Wijnen, A.P. Direcks, Current guidelines for gynecological examination in des daughters[dutch] Ned. Tijdschr. Geneeskd. 136 (1992) 2065-2067.

[22] D.A. Antonioli, L. Burke, Vaginal adenosis. Analysis of 325 biopsy specimens from 100 patients, Am. J. Clin. Pathol. 64 (1975) 625-638.

[23] J.A. Jefferies, S.J. Robboy, P.C. O'Brien, E.J. Bergstralh, D.R. Labarthe, A.B. Barnes, et al., Structural anomalies of the cervix and vagina in women enrolled in the diethylstilbestrol Adenosis (DESAD) project, Am. J. Obstet. Gynecol. 148 (1984) 59-66.

[24] M. Tournaire, E. Devouche, S. Epelboin, A. Cabau, Diethylstilbestrol exposure: evaluation of the doses received in France, Eur. J. Epidemiol. 27 (2012) 315-316.

[25] Comparison of risk factors for invasive squamous cell carcinoma and adenocarcinoma of the cervix: collaborative reanalysis of individual data on 8,097 women with squamous cell carcinoma and 1,374 women with adenocarcinoma from 12 epidemiological studies, Int J Cancer 120 (2007) 885-891.

[26] M. Schiffman, N. Wentzensen, S. Wacholder, W. Kinney, J.C. Gage, P.E. Castle, Human papillomavirus testing in the prevention of cervical cancer, J. Natl. Cancer Inst. 103 (2011) 368-383.

[27] M. Kocken, A. Baalbergen, P.J Snijders, J. Bulten, W.G. Quint, F. Smedts, et al., Highrisk human papillomavirus seems not involved in DES-related and of limited importance in nonDES related clear-cell carcinoma of the cervix, Gynecol. Oncol. 122 (2011) 297-302

[28] D.D. Baird, A.J. Wilcox, A.L. Herbst, Self-reported allergy, infection, and autoimmune diseases among men and women exposed in utero to diethylstilbestrol, J. Clin. Epidemiol. 49 (1996) 263-266.

[29] L. Burke, M. Segall-Blank, C. Lorenzo, R. Dynesius-Trentham, D. Trentham, J.F. Mortola, Altered immune response in adult women exposed to diethylstilbestrol in utero, Am. J. Obstet. Gynecol. 185 (2001) 78-81. 